\title{
Combined SEM-FIB-SPM-TOF-EDX-EBSD as a Multifunctional Tool
}

J. Jiruše ${ }^{1}$, L. Sedláček ${ }^{1}$, M. Rudolf ${ }^{1}$, V. Friedli ${ }^{2}$, F. Oestlund ${ }^{3}$, J. Whitby ${ }^{4}$

${ }^{1}$ TESCAN a.s., Libušina tř́da 21, 62300 Brno, CZECH REPUBLIC

${ }^{2}$ SPECS Surface Nano Analysis GmbH, Voltastrasse 5, 13355 Berlin, GERMANY

${ }^{3}$ TOFWERK AG, Uttigenstrasse 22, 3600 Thun, SWITZERLAND

${ }^{4}$ EMPA, Feuerwerkerstrasse 39, 3602 Thun, SWITZERLAND

Recent rapid advances in nanotechnology leads to a growing need for a powerful "multi-nano tool" capable of nano-manipulation, nano-structuring (i.e. surface modification), nano-imaging and nanoanalysis in a single instrument. Such a novel tool is prepared with a wide variety of techniques. Vision capability is ensured by Scanning Electron Microscope (SEM) facility combined with Scanning Probe Microscopy (SPM). Structuring capability is obtained using Focused Ion Beam (FIB). One can choose from three different FIB-columns. Manipulation add-ons use either SPM facility or specially designed end-effectors. A real strength of the new instrument lies in its variety of nano-analytic tools. A new dedicated chamber with more than 20 ports integrates Energy Dispersive X-ray Spectrometer (EDX), Electron Back-Scatter Diffraction (EBSD), Time-of-Flight Mass Spectrometry (TOF-SIMS), electron Beam Induced Current (EBIC) or Cathodoluminescence facilities. All these tools fit to the common analytical working distance, thus the sample does not need to be moved between experiments. Combined SEM-FIB-SPM-TOF-EDX-EBSD instrument gives new possibilities for nanotechnology.

This extensive integration contains three main topics: integration of SPM with dual FIB-SEM, integration of TOF-SIMS with dual FIB-SEM and complementary use of various techniques to obtain 3D tomography.

In the first topic, a scanning probe microscope from SPECS able to work in STM, AFM or MFM modes was integrated with a combined focused ion and scanning electron microscope from TESCAN. The compact SPM can be operated in the intersection of electron and ion beams thus a simultaneous use of all three techniques is possible. This is accomplished without affecting any detectors or analyzers present in the combined SEM-FIB chamber.

The integrated SPM-SEM-FIB offers many advantages like simultaneous SPM and SEM imaging, real-time navigating of the SPM head using SEM, depth profiling by SPM for freshly prepared structures by FIB, fast inspection of SPM tip quality by SEM and so on. The SPM in vacuum also does not suffer from the water cavity effect arising in the air.

A unique combination of orthogonal TOF-SIMS with FIB and SEM was realized for the first time [1]. It provides a cost-effective upgrade to dual SEM-FIB. It allows secondary ion imaging as well as depth profiling and so full three-dimensional chemical images with nanometer resolution can be obtained. TOF-SIMS gives elemental, isotopic and also chemical information about the sample. A compact TOF-SIMS module with orthogonal extraction is an add-on to a standard high vacuum Gallium FIB, thus no other primary ion source is needed. Chemical images are achieved with better than $50 \mathrm{~nm}$ lateral and $20 \mathrm{~nm}$ depth resolution. User is benefited by all advantages of combined SEM-FIB (imaging, etching, deposition, lithography, manipulation) and can perform SIMS analysis in-situ just after preparation of desired nanostructures. 
A variety of other techniques allow a 3D tomography approach based on sequential FIB slicing followed by analysis and subsequent data-processing to create $3 \mathrm{D}$ objects with analytical information. The visualization can choose from all main methods like cross sections, iso surfaces and direct volume rendering. Practical examples are given by $3 \mathrm{D}$ BSE reconstruction of a microprocessor or 3D EBIC reconstruction of silicon nanowires based solar cell [2]. In the last example, the scanning electron beam generates charge carrier pairs that can be separated by the junction. The beam position combined with the flowing current gives a map of the active region. The clear EBIC-signal from the wire shows that charge carriers, once generated in the wires by absorbing light, can be separated by the junction. This experiment shows that silicon nanowires are active photovoltaic components and not only passive antireflective structures.

\section{References:}

[1] Whitby J.A., et al., "High Spatial Resolution Time-of-Flight Secondary Ion Mass Spectrometry for the Masses: A Novel Orthogonal ToF FIB-SIMS Instrument with In Situ AFM", Adv. Mat. Sci. Eng. 2012.

[2] Sivakov V., et al., "Wet - Chemically Etched Silicon Nanowire Architectures: Formation and Properties", Nanowires - Fundamental Research, Abbass Hashim (Ed.), ISBN: 978-953-307-327-9, InTech.

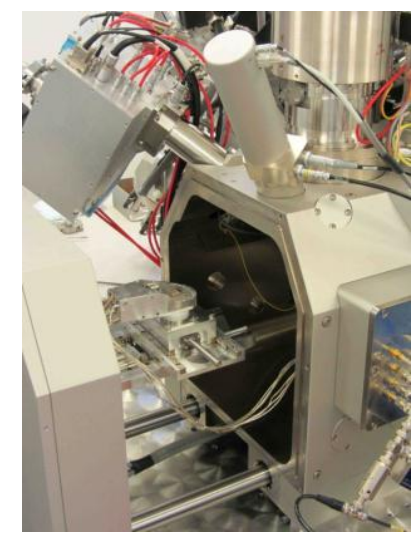

Fig. 1. SPM (on stage) and TOF (top left) integrated into FIB-SEM.
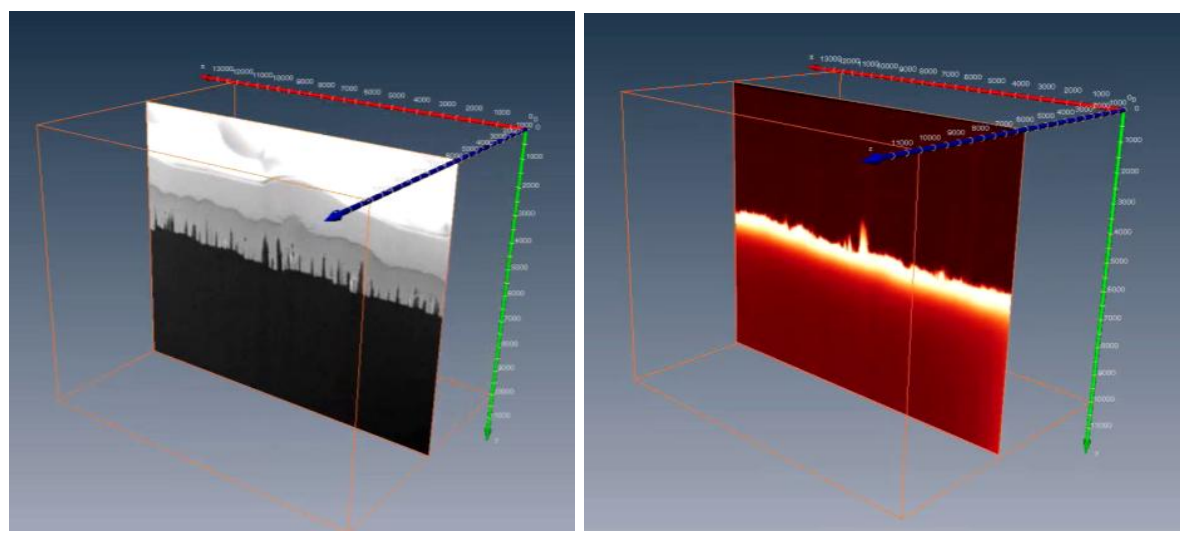

Fig. 2. Screenshots from a cross-sectional movie of the slicing process of silicon nanowires based solar cell. Comparison of SE signal (left) and EBIC signal (right) gives the information about active nanowire. 